\title{
PHENOMENON OF REMOTE INTERACTION AND SORPTION ABILITY OF RARE CROSS-LINKED HYDROGELS OF POLYMETHACRYLIC ACID AND POLY-4-VINYLPYRIDINE IN RELATION TO ERBIUM IONS
}

\author{
Talkybek Jumadilov ${ }^{1}{ }^{凶}$, Ruslan Kondaurov ${ }^{1}$, Aldan Imangazy $^{1}$, \\ Nurbala Myrzakhmetova1, Indira Saparbekova ${ }^{1}$
}

https://doi.org/10.23939/chcht13.04.451

\begin{abstract}
Phenomenon of the long-range effect of poly(methacrylic acid) hydrogel - poly(4-vinylpyridine) hydrogel (gPMAA-gP4VP intergel system) on erbium ions sorption was studied. It was established that the structure of the basic hydrogel had a significant impact on the self-organization of the PMAA hydrogel. The mutual activation of hydrogels was investigated in an aqueous medium. Dependencies of swelling coefficient, specific electric conductivity and $\mathrm{pH}$ of aqueous solutions were determined. Individual PMAA and P4VP hydrogels showed the erbium ions extraction degree of about $44 \%$ and $17 \%$, respectively. At the ratios $5: 1 \quad(83 \%$ of gPMAA-17\% gP4VP) and 4:2 (67\% gMPAA-33\% gP4VP), $56 \%$ and $53 \%$ of erbium ions were recovered, respectively. After $48 \mathrm{~h}$ the PMAA and P4VP hydrogels had the polymer chain binding degree of $70 \%$. The obtained results point to the evidence that at these ratios there are significant changes in electrochemical and conformational degrees of initial macromolecules in gPMAA-gP4VP intergel system.
\end{abstract}

Keywords: intergel system, polymethacrylic acid, poly-4vinylpyridine, remote interaction, $\mathrm{Er}^{3+}$ ions, sorption.

\section{Introduction}

Rare-earth metals (REM) are used in various industries: chemical industry, nuclear engineering, radio electronics, machine building, metallurgy, etc. These elements and their compounds are widely distributed in the chemical industry, for example, in the production of varnishes, pigments and paints, and in the oil industry as

\footnotetext{
${ }^{1}$ Institute of Chemical Sciences after A.B. Bekturov, 106, Sh. Valikhanov St., 050010 Almaty, Republic of Kazakhstan jumadilov@mail.ru

(c) Jumadilov T., Kondaurov R., Imangazy A., Myrzakhmetova N., Saparbekova I., 2019
}

catalysts. REM are used in the production of special steels, alloys (as degasifiers), and certain explosives [1-3]. Single-crystal compounds based on REM are used in creation of laser and other optically active elements in optoelectronics [4]. Based on elements such as erbium, neodymium, yttrium, samarium alloys with recordable magnetic properties (high magnetizing and coercive forces) are produced to create permanent magnets of enormous power in comparison with simple ferroalloys [5]. Previous studies [6-9] have shown that mutual activation of polymer hydrogels leads to significant changes in their conformational and electrochemical properties. Remote interaction is carried out by rapid hydration, ionization, dissociation, association and slow change in the conformational state of inter-node links. It is obvious that the initial dry state of the rare-crosslinked hydrogels should influence the rate of interactions in the intergel system. As can be seen from Fig. 1, intergel system consists of two hydrogels, the remote interaction of which occurs in joint solution. Swollen polymers are prone to mutual activation due to unfolding of the macromolecular globe during swelling, therefore, the activity of functional groups increases. Relating to this, the purpose of this work was also to study the sorption ability of the gPMAA-gP4VP intergel system consisting of preliminary swollen hydrogels in relation to erbium ions.

Polymer hydrogels usually are considered as smart materials, and their progressive functions are developing. In particular, different biomimetics and membrane applications are discussed widely in current literature [10-12].

Interactions between different types of hydrogels are much less investigated than hydrogels themselves; one can find only individual reports on this research $[9,13]$. Besides, cited articles $[9,13]$ are devoted to contact interactions of hydrogels. 


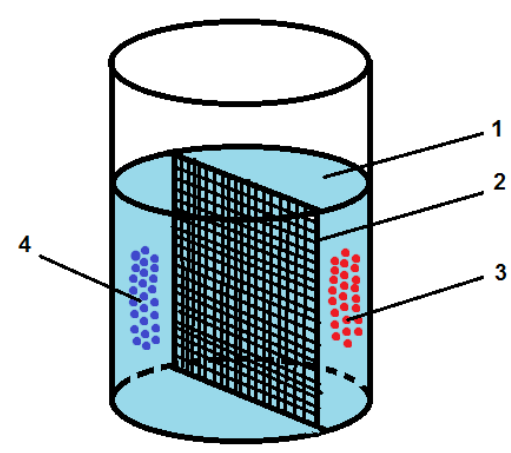

Fig. 1. Intergel system: 1 - solution, 2 - polypropylene membrane, 3 - acid hydrogel, 4 - base hydrogel. Intergelic systems - multicomponent systems which consist of two or more hydrogels and solution (there is no direct contact between polymers)

Now we report about sufficiently new phenomena, which demonstrate possibility of electrochemical interactions between samples placed at quite long distance from each other. The organic chemical reaction directly resulting in charging of a macroscopic specimen is observed. Non-zero macroscopic charge appears during long-distance interactions between two polymer hydrogels, when one network is a cross-linked poly-acid, which generates moveable hydrogen ions, and another cationic hydrogel can take part in a chemical reaction of bonding of the mentioned ions [14].

\section{Experimental}

For measuring the specific electric conductivity of aqueous solution, conductometer MARK-603 (Russia) was used; the hydrogen ions concentration was determined by pH-meter Metrohm 827 pH-Lab (Switzerland). The mass of samples was estimated using analytical scales SHIMADZU AY220 (Japan). Optical density measurements for the subsequent calculation of the erbium nitrate concentration were determined by spectrophotometers Perkin Elmer Lambda 35 (USA) and Jenway-6305(UK).

\subsection{Materials}

This research was carried out in an erbium(III) nitrate pentahydrate solution. The hydrogels of poly(methacrylic acid) were synthesized in the presence of the cross-linking agent $N, N$-methylene-bis-acrylamide and the oxidation-reduction system $\mathrm{K}_{2} \mathrm{~S}_{2} \mathrm{O}_{8}-\mathrm{Na}_{2} \mathrm{~S}_{2} \mathrm{O}_{3}$. The poly(4-vinylpyridine) hydrogel (gP4VP) was synthesized by Sigma-Aldrich ( $2 \%$ cross-linking agent). The synthesized hydrogels in the aqueous medium constituted the intergel pair "poly(methacrylic acid) hydrogel - poly(4-vinylpyridine) hydrogel" (gPMAA-gP4VP). The hydrogels swelling degrees were: $\alpha(\mathrm{gPMAA})=20.65 \mathrm{~g} / \mathrm{g}$; $\alpha(\mathrm{gP} 4 \mathrm{VP})=2.65 \mathrm{~g} / \mathrm{g}$.

\subsection{Electrochemical Investigations}

Experiments were carried out at room temperature. The studies of intergel system were made in the following order: (i) each hydrogel in a dry initial state was put in polypropylene mesh and placed in a glass with distilled water for activation (filter pores of polypropylene mesh was permeable for low molecular ions but non-permeable for hydrogel dispersion; (ii) these filters with hydrogels were then put into glasses with aqueous and erbium nitrate solutions; (iii) electric conductivity and $\mathrm{pH}$ of the solutions were measured in the presence of hydrogels.

\subsection{Determination of Hydrogels Swelling}

Swelling coefficient was calculated by Eq. (1):

$$
K_{s w}=\frac{m_{2}-m_{1}}{m_{1}}
$$

where $m_{1}$ and $m_{2}$ are masses of dry and swelled hydrogels, respectively, $\mathrm{g}$.

\subsection{Methodology of Erbium Ions Determination}

Methodology of erbium ions determination based on formation of colored complexed compound of organic analytic reagent Arsenazo III with erbium ions [13].

Total binding degree of internode links of polymer chain was calculated according to Eq. (2):

$$
\theta=\frac{v_{\text {sorp }}}{v} \cdot 100 \%
$$

where $v_{\text {sorp }}$ and $v$ are quantities of sorbed erbium and polymer sample, respectively, mol. If there are 2 hydrogels in solution, it is calculated as sum of quantity of each of them.

\section{Results and Discussion}

\subsection{Study of Mutual Activation of gPMAA and gP4VP Hydrogels in Intergel system. Long-distance \\ Interactions Mechanism}

The erbium ions sorption by the gPMAA-gP4VP intergel system occurs by ionic and coordination mechanisms. 
Two main reactions occur in intergel systems: 1. Acid hydrogel dissociation:

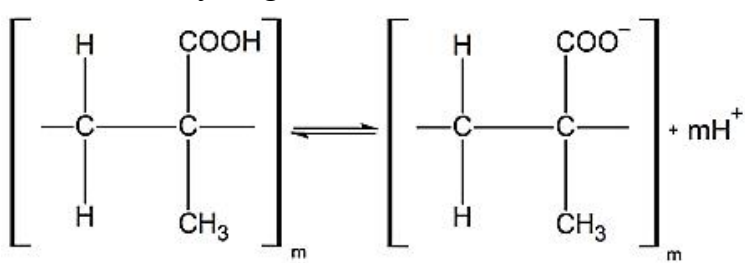

2. Binding of cleaved proton by nitrogen heteroatom:<smiles>ICC(I)c1ccncc1</smiles>

Dissociation occurs stepwise: firstly, there is ionization with ionic pair's formation, after that ionic pairs partially dissociate to separate ions.

Ionization and partial dissociation of nitrogen heteroatom in pyridine ring:

$$
\equiv \mathrm{N}+\mathrm{H}_{2} \mathrm{O} \rightarrow \equiv \mathrm{NH}^{+} \ldots \mathrm{OH}^{-} \rightarrow \equiv \mathrm{NH}^{+}+\mathrm{OH}^{-}
$$

Interaction of nitrogen heteroatom with proton, which was cleaved from carboxyl group:

$$
\equiv \mathrm{N}+\mathrm{H}^{+} \rightarrow \equiv \mathrm{NH}^{+}
$$

Water molecules formation by interaction of $\mathrm{H}^{+}$and $\mathrm{OH}^{-}$ions formed after functional groups reaction with water molecules:

$$
\mathrm{H}^{+}+\mathrm{OH}^{-} \rightarrow \mathrm{H}_{2} \mathrm{O}
$$

Typically, reactions (3) and (4) do not break up the electric neutrality of a system, but in our case, the reaction (3) takes place in volume of one specimen, and the other occurs in volume of sample which is placed at relatively long distance. Generated hydrogen ions in the volume of the first sample are chemically bonding by neighboring specimen. In other words, poly(methacrylic acid) gel (reaction 3) may be considered as a donor and the second gel as an acceptor of protons (reaction 4). Consequently, both gels get non-zero electrostatic charge (Fig. 2), and behavior of the system now is determined by macroscopic charge distribution.

First, non-zero charge of each specimen leads to repulsion of charged functional groups of each gel from each other. It is necessary to highlight that functional groups charges inside each gel actually are moveable. Their motion has the same mechanism as holes conductivity in semiconductors. A proton may disconnect from a $-\mathrm{COOH}$ group and join the neighbor dissociated ${ }^{-} \mathrm{COO}^{-}$group. Totally, such process may be considered as motion of $-\mathrm{COO}^{-}$charged "particle". Repulsion of such negative particles leads to formation of a thin charged layer at surface of a specimen, which take part in longdistance interaction with acceptor of protons, while the degree of dissociation of weak carbon acid is low. The erbium ions sorption by the gPMAA-gP4VP intergel system occurs by ionic and coordination mechanisms.

Under condition of electric neutrality of a system as a whole and its macroscopic parts separately, electrochemical processes usually occur. A set of electrochemical reactions may be quite complicated, but the deviations from neutrality usually have scale determined by Debye length only, i.e. known types of chemical processes cannot result in appearance of macroscopic electric charge and electric currents [14].

High sorption degree of polymers in intergel systems is due to the absence of counter ions at ionized groups. This consequence of intergel interactions results in mutual activation of hydrogels and uncompensated charges formation along the polymer chain. Uncompensated charge is formed due to cleavage of proton from carboxyl groups during dissociation of acid hydrogel and its binding with heteroatom of polybasis in an aqueous medium, wherein charge density of basic hydrogels is limited by dissociation degree of acid hydrogel. Subsequently, both hydrogels are ionized and do not have counter ions at major part of functional groups. Ionized groups formation is caused by conformational changes of polymer chains internode links. Due to these conformational changes, unfolding of macromolecular globe occurs.

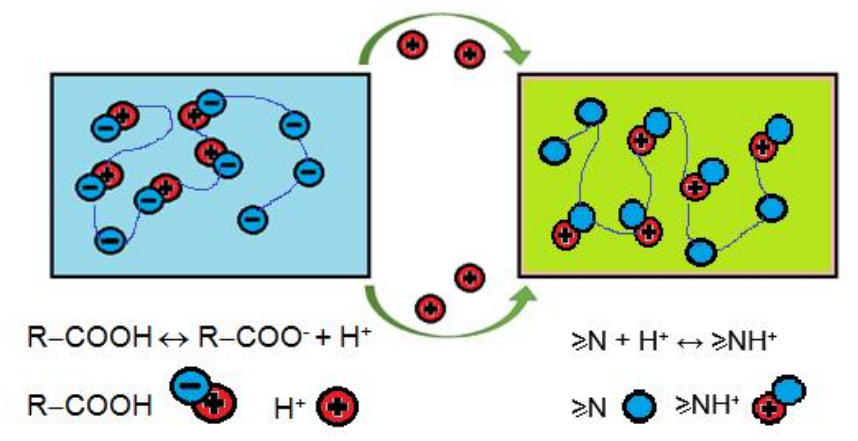

Fig. 2. Formation of macroscopic charge at long-distance interaction between acceptor and donor networks 
Uncompensated charge is formed due to proton cleaving from carboxyl group during acid hydrogel dissociation and association of this ion by heteroatom of basic hydrogel in an aqueous medium. In this case charge density of basic hydrogels is limited by dissociation degree of acid hydrogel. Consequently, both hydrogels are ionized and do not have counter ions at charged links. The result is an increased sorption ability in comparison with individual hydrogels [14].

\subsection{Study of the Electrochemical, Volumetric-Gravimetric Properties of the Poly(methacrylic acid) Hydrogel}

The next goal of our research was to study the impact of dry initial state of PMAA and P4VP polymer hydrogels on electrochemical and volume-gravimetric properties of gPMAA-gP4VP intergel system. Feature of ionization process in intergel systems is in the absence of counter ions at ionized groups. This is the consequence of intergelic interactions, and, consequently, there is an occurrence of hydrogels mutual activation and uncompensated charges formation along the polymer chain.

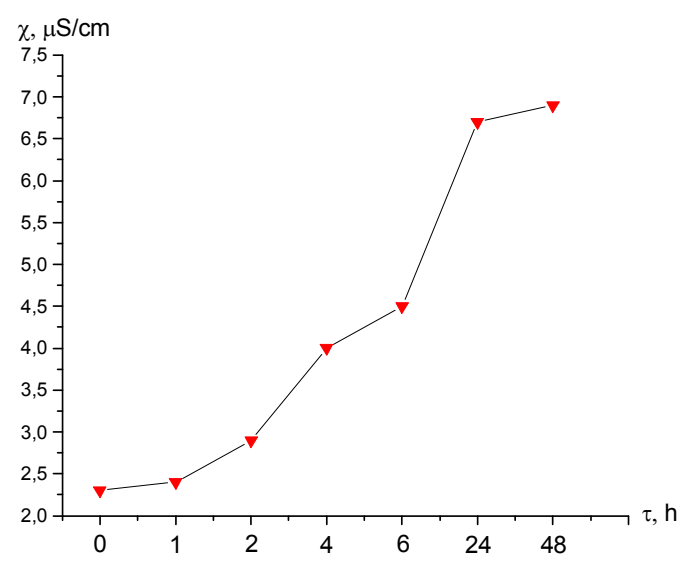

Fig. 3. Kinetics of the electrical conductivity change in the presence of the PMAA hydrogel

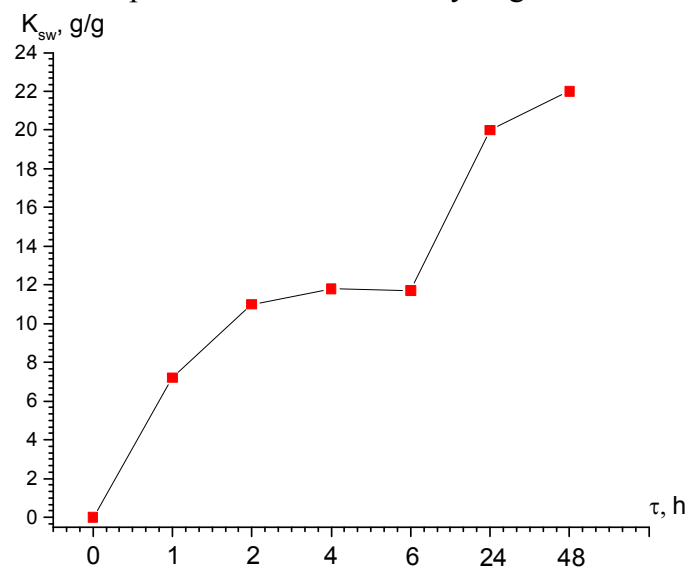

During the interaction of poly(methacrylic acid) hydrogel with an aqueous medium, various changes in the electrochemical properties of water occur. Fig. 3 demonstrates the dependence of the specific electrical conductivity of the aqueous solution on time in the presence of the PMAA hydrogel. The dependence curve clearly shows that the electrical conductivity values increase with time. A strong increase occurs in the first 6 hours of interaction of the poly(methacrylic acid) hydrogel with the solution. This is facilitated by the dissociation of functional groups on the inter-node links of the macromolecule. After that, up to $48 \mathrm{~h}$, there is practically no increase in the electrical conductivity, which in turn leads to the conclusion that the system has reached a state of equilibrium.

Then, there is an intensive growth of $\mathrm{pH}$ up to $24 \mathrm{~h}$. The main reason for these phenomena is the decomposition of associates within the macromolecule, followed by the addition of protons to carboxylate-formed anions. It should be noted that in this case intramolecular associates are stronger than in the structure of polyacrylic acid. This is due to the higher degree of hydrophobicity of the poly(methacrylic acid) hydrogel.

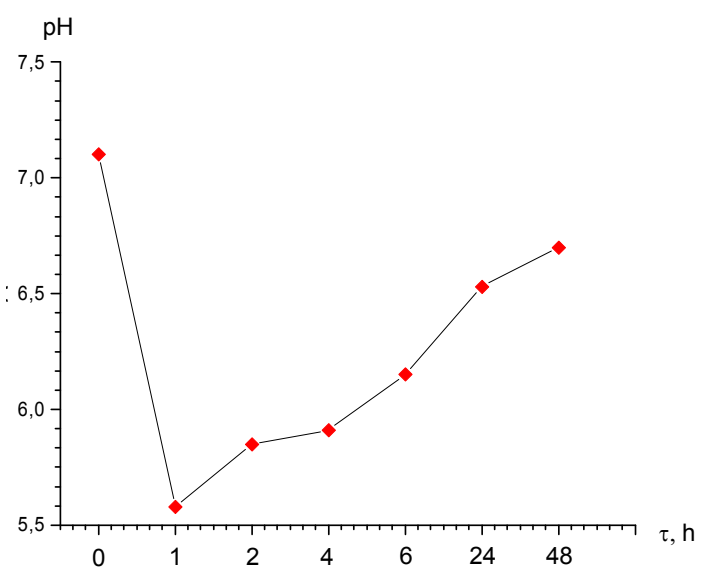

Fig. 4. Kinetics of $\mathrm{pH}$ change in the presence of PMAA hydrogel

Fig. 5. Kinetics of the PMAA hydrogel swelling ratio change 
Fig. 4 shows the change in $\mathrm{pH}$ at various times in the presence of PMAA hydrogel. At the initial time, a significant decrease in the $\mathrm{pH}$ value occurs, indicating that protons are released into the solution upon dissociation of the carboxyl groups.

Fig. 5 demonstrates the dependence of the swelling coefficient of the poly(methacrylic acid) hydrogel. As can be seen from the figure, a sharp increase in swelling is observed during $1 \mathrm{~h}$. This is due to the functional carboxyl groups dissociation and the unfolding of the polymer globe. Less intensive increase is observed within 6 hours. Further, the increase in the swelling coefficient occurs slowly, which allows one to conclude that equilibrium in the system is reached.

\subsection{Study of the Electrochemical and Volumetric-Gravimetric Properties of the Poly(4-vinylpyridine) Hydrogel}

The dependence of the specific electrical conductivity of the aqueous solution on time in the presence of poly(4-vinylpyridine) hydrogel is shown in Fig. 6 . The obtained results indicate that the conductivity values decrease with time. This is due to the fact that the

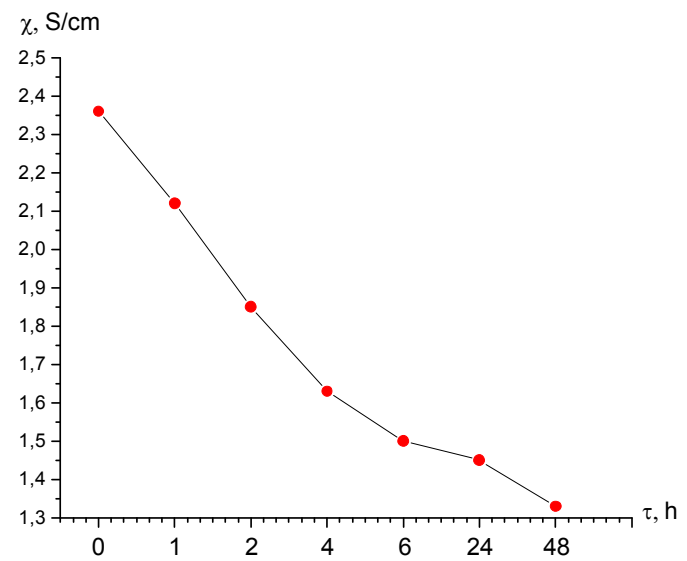

Fig. 6. Kinetics of the electrical conductivity change in the presence of the P4VP hydrogel

Fig. 8. Kinetics of the P4VP hydrogel swelling ratio change binding of protons by nitrogen heteroatoms of vinyl pyridine occurs and $\mathrm{H}^{+}$is formed as a result of water molecules dissociation. As can be seen from Fig. 6, a significant reduction occurs in $6 \mathrm{~h}$ after the initiation of the polybase interaction with the aqueous solution. Then, there is a slight decrease in electrical conductivity up to $48 \mathrm{~h}$.

Fig. 7 shows the dependence of the hydrogen ion concentration at various durations. As can be seen from Fig. 7, at the initial moment of time, a gradual increase in the proton concentration occurs, then, their content in the solution decreases, indicating that the proton is associated with a polybase. At the initial instant of time, the polybase, as evidenced by the high values of their concentration, weakly binds the protons. Then, after $6 \mathrm{~h}$, the degree of their association increases, and the concentration of hydrogen ions in the solution decreases. This leads to the increase in the medium $\mathrm{pH}$.

Swelling of poly(4-vinylpyridine) hydrogel occurs rather intensively during $1 \mathrm{~h}$, then swelling decreases. The main reason for the decrease in swelling can be the folding of the macromolecule polymer globe. This is due to the intramolecular cross-links formation, such as:

$$
\geq \mathrm{N} \ldots \mathrm{H}+\ldots \mathrm{N} \equiv
$$

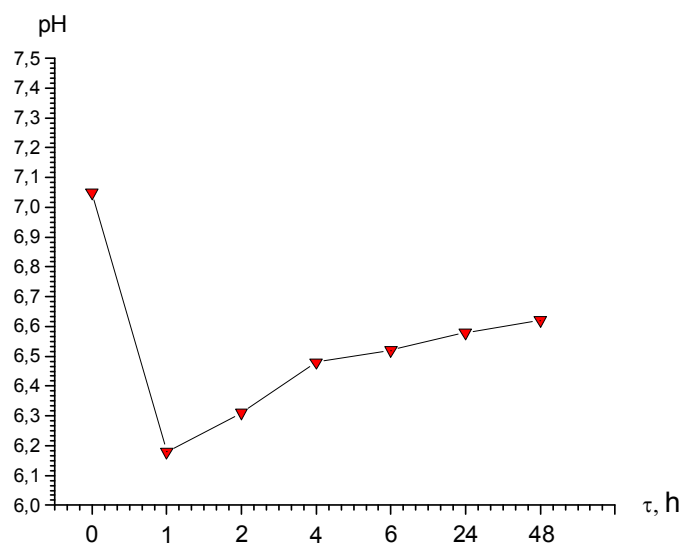

Fig. 7. Kinetics of $\mathrm{pH}$ change in the presence of P4VP hydrogel

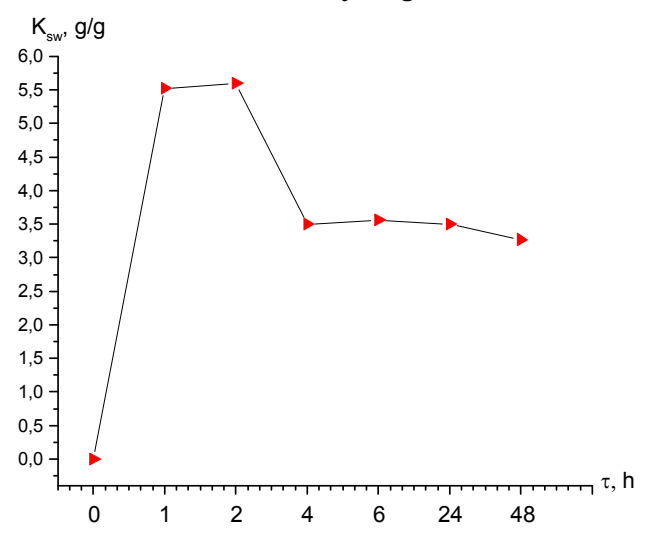


As can be seen from Fig. 8, after $24 \mathrm{~h}$ the swelling coefficient remains practically unchanged. In other words, we can say that the state of equilibrium is reached.

In the process of hydrogelsremote interaction in the gPMAA-gP4VP intergel system, the specific electric conductivity of aqueous solutions changes. The dependence of the specific electrical conductivity on the molar ratio of hydrogels in time is shown in Fig. 9. As can be seen from the image, the increase in electrical conductivity occurs at the ratio of gPMAA:gP4VP $=3: 3$ over the entire time of the remote interaction. The maximum electrical conductivity was reached after $48 \mathrm{~h}$. The minimum values of the electrical conductivity are noted in the region of only polybase presence (gMPAA:gP4VP $=0: 6$ ), which is due to its weak dissociation.

The dependence of the hydrogen ions concentration is shown in Fig. 10. As can be seen from Fig. 10, the increase in the hydrogen ions concentration occurs at the ratio of gPMAA:gP4VP $=3: 3$. If we compare these data

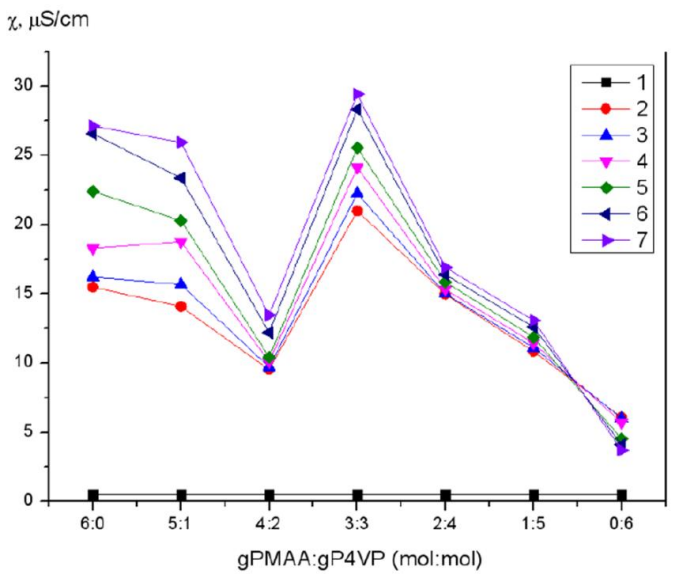

Fig. 9. Dependence of the specific electrical conductivity of aqueous solutions on the mole content of hydrogels in time: $0 \mathrm{~h}$ (1); $0.5 \mathrm{~h} \mathrm{(2);1} \mathrm{h} \mathrm{(3);} 2 \mathrm{~h} \mathrm{(4);6} \mathrm{h} \mathrm{(5);} 24 \mathrm{~h} \mathrm{(6)} \mathrm{and} 48 \mathrm{~h}$ (7)

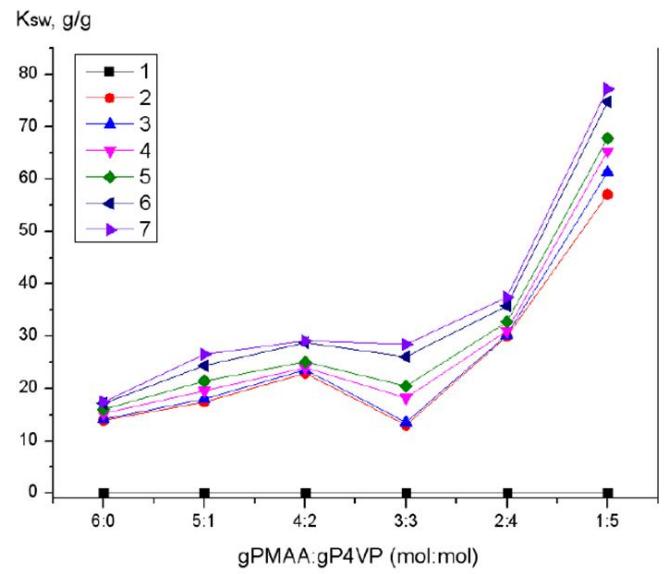

Fig. 11. Dependence of the swelling ratio of gPMAA in the presence of gP4VP on the molar content of hydrogels in time:

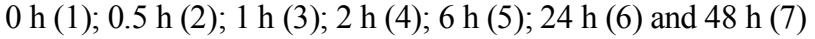

with the data on the electrical conductivity, then we can conclude that at this ratio, the process of carboxyl groups dissociation predominates over the process of attaching protons to vinylpyridine.

A significant decrease in the concentration of $\mathrm{H}^{+}$ ions occurs at the ratio of gPMAA:gP4VP $=1: 5$. In the case of specific electric conductivity, at the same ratio, it is clear that the electrical conductivity values are low. The result of this is the formation of the same charged functional groups without counterions. As a consequence - the transition of acid and basic hydrogels into a highly ionized state takes place.

Fig. 11 demonstrates the dependence of the swelling coefficient of the acid hydrogel poly(methacrylic acid) on the molar ratio of hydrogels in the gPMAA:gP4VP intergel system over time. The increase in the swelling coefficient of the polyacid occurs gradually with time increasing.

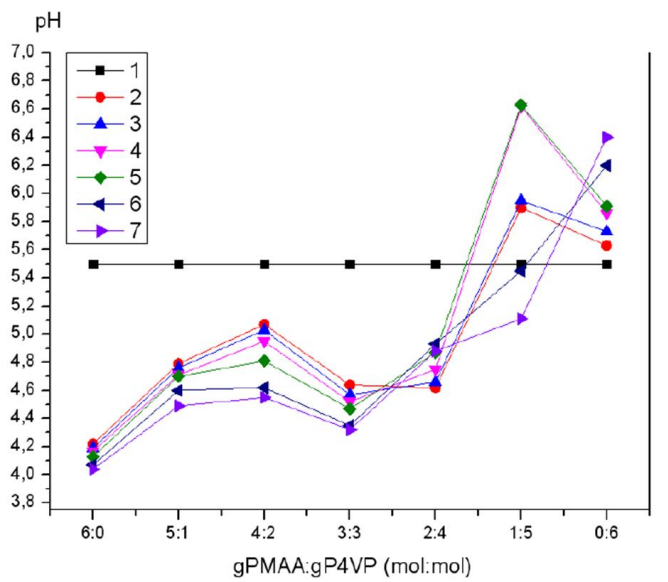

Fig. 10. Dependence of aqueous solutions $\mathrm{pH}$ on the mole content of hydrogels in time: $0 \mathrm{~h} \mathrm{(1);0.5} \mathrm{h} \mathrm{(2);} 1 \mathrm{~h} \mathrm{(3);} 2 \mathrm{~h} \mathrm{(4);}$

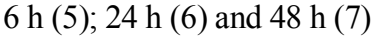

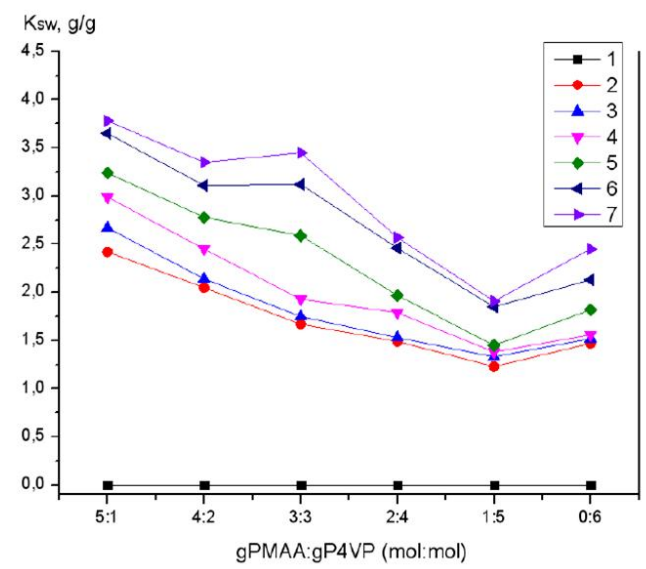

Fig. 12. Dependence of the P4VP swelling ratio in the presence of gPMAA on the molar content of hydrogels in time: $0 \mathrm{~h}(1)$;

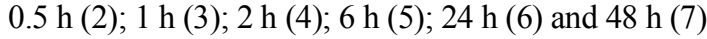


Moreover, it should be noted that the increase in $K_{s w}$ occurs with an increase in the concentration of the polybase in the solution. The minimum swelling occurs in the presence of only polyacid in the solution (gPMAA:gP4VP $=6: 0$ ). The maximum swelling of the poly(methacrylic acid) hydrogel occurs at the ratio of gPMAA:gP4VP $=1: 5$ after $48 \mathrm{~h}$ of the hydrogels remote interaction. This is due to the predominance of the proton association process over the dissociation of carboxyl groups. The result of this is the transition of the acid hydrogel into a highly ionized state.

Fig. 12 shows the dependence of the swelling coefficient of the main hydrogel poly(4-vinylpyridine) on the mole ratio of hydrogels in the gPMAA:gP4VP intergel system over time.

As the percentage of polyacid increases, the swelling coefficient of the polybase increases. The minimum swelling of the poly-base occurs at the ratio of gPMAA:gP4VP $=1: 5$. This is due to the formation of intramolecular cross-links $\geq \mathrm{N} \ldots \mathrm{H}+\ldots \mathrm{N} \equiv$, which results in folding of the polymer globe and a decrease in swelling. The maximum swelling area is the ratio of gPMAA:gP4VP $=5: 1$, with $\mathrm{KH}$ taking the maximum values at this ratio after $48 \mathrm{~h}$.

\subsection{Study of Erbium Ions Extraction by gPMAA-gP4VP Intergel System}

The dependence of erbium ions extraction degree by the gPMAA-gP4VP intergel system on the mole ratio of hydrogels in time is shown in Fig. 13.

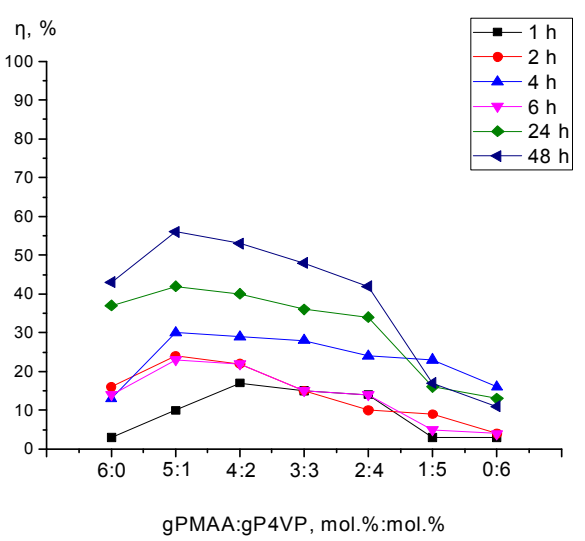

Fig. 13. The dependence of the erbium ions extraction degree by the gPMAA-gP4VP intergel system on the mole ratio of hydrogels in time

The maximum amount of erbium ions was extracted by the gPMAA-gP4VP intergel system at the ratio of hydrogels 5:1 (83\% gMPAA-17\% gP4VP) and $4: 2$ (67\% gPMAA-33 \% gP4VP). The degree of erbium ions extraction after $48 \mathrm{~h}$ at these ratios was 56 and $53 \%$, respectively. The obtained results indicate that the degree of erbium ions extraction by individual hydrogels of poly(methacrylic acid) and poly(4-vinylpyridine) is low, the extraction rates were 44 and $17 \%$, respectively. The remaining ratios of the intergel pair in the gPMAAgP4VP intergel system also have much higher erbium ion extraction rates compared to the erbium ions extraction of the PMAA and P4VP individual hydrogels.

\subsection{Study of the Polymer Chain Binding Degree of the gPMAA-gP4VP Intergel System}

Fig. 14 shows the dependence of the polymer chain binding degree (with respect to erbium ions) of the gPMAA-gP4VP intergel system in time. The maximum values of the polymer chain binding degree in the intergel system after $48 \mathrm{~h}$ were $0.7 \%$ at the ratios of gPMAAgP4VP 5:1 and 4:2. The polymer chain binding degree values of poly(methacrylic acid) and poly(4-vinylpyridine) individual hydrogels after $48 \mathrm{~h}$ were 0.54 and $0.13 \%$, respectively. After $2 \mathrm{~h}$ of sorption, a certain increase in the polymer chain binding degree was achieved. For 24 and $48 \mathrm{~h}$, a significant increase in the polymer chain binding degree was observed; this is particularly noticeable for $4: 2$ and 3:3 ratios.

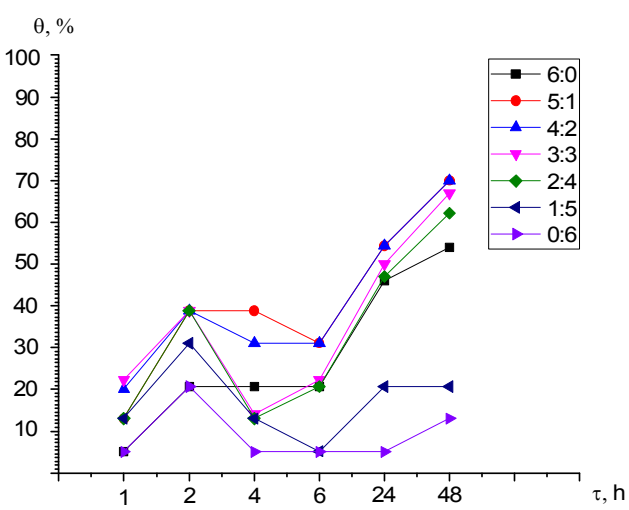

Fig. 14. The dependence of the polymer chain binding degree of the gPMAA-gP4VP intergel system on time in the erbium(III) nitrate pentahydrate medium

\subsection{Study of the Effective Dynamic Exchange Capacity of the gPMAA- gP4VP Intergel System}

Fig. 15 shows the dependence of the effective dynamic exchange capacity of the gPMAA-gP4VP intergel system on the molar ratio of hydrogels in time. The obtained data indicate that the mutual activation of polymer hydrogels in the intergel pair leads to a significant increase in the values of the exchange capacity in comparison with the individual hydrogels. The maximum value of the effective dynamic exchange capacity was achieved at gPMAA-gP4VP ratio of $5: 1$ for $48 \mathrm{~h}$ of hydrogels 
remote interaction. Further remote interaction of polymer hydrogels indicates that the gPMAA-gP4VP intergel system approaches the equilibrium state, which is evidenced by slower growth of the effective dynamic exchange capacity as at the beginning of the hydrogels remote interaction.

The following ions are present in the solution: $-\mathrm{COO}^{-}, \mathrm{H}^{+}, \mathrm{Er}^{3+}$ and $\mathrm{NO}_{3}^{-}$. There is an occurrence of these chemical reactions in solution:

1. Dissociation of erbium nitrate along with carboxyl groups dissociation:

$$
\begin{aligned}
& -\mathrm{COOH} \leftrightarrow-\mathrm{COO}^{-}+\mathrm{H}^{+} \\
& \mathrm{Er}\left(\mathrm{NO}_{3}\right)_{3} \cdot 5 \mathrm{H}_{2} \mathrm{O} \leftrightarrow \mathrm{Er}^{3+}+3 \mathrm{NO}_{3}{ }^{-}+5 \mathrm{H}_{2} \mathrm{O}
\end{aligned}
$$

2. Sorption oh erbium ions by polymer hydrogels:

$3-\mathrm{COO}^{-}+\mathrm{Er}^{3+} \rightarrow-\mathrm{COO}_{3} \mathrm{Er}$

$3 \equiv \mathrm{N}+\mathrm{Er}^{3+} \rightarrow \equiv \mathrm{N}_{3}^{+} \mathrm{Er}$

Electrochemical equilibrium in solution depends on these reactions and there could be changes of electrical conductivity values in dependence of dominance of any one of them.

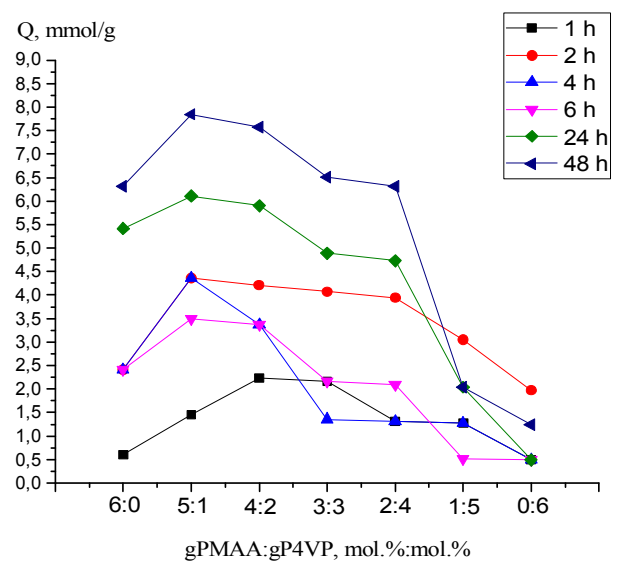

Fig. 15. The dependence of the effective dynamic exchange capacity of the gPMAA-gP4VP intergel system on the mole ratio of hydrogels in time

\section{Conclusions}

The obtained results allow to make the following conclusions. Polymer macromolecules mutual activation leads to significant changes in electrochemical and conformational properties of hydrogels. Based on the data of conductivity, $\mathrm{pH}$ and swelling coefficient it can be concluded that erbium ions sorption by polymer hydrogels occurs. Maximum total binding degree of polymer chain $(70 \%)$ was observed at gPMAA:gP4VP ratio of $4: 2$. Mutual activation by gPMAA and gP4VP hydrogels in intergel system provides higher sorption degree for intergel pair as compared to individual hydrogels.

Hydrogels long-range effect (Jumadilov's effect) may be a basis for creation of innovative technologies in hydrometallurgy, wastewater treatment, industrial and biological solutions, and in other areas where the necessity in selective separation, concentration and extraction of different charged particles is urgent.

\section{References}

[1] Alimbekova B., Erzhet B., Korganbayeva Zh. et al.: Proceed. VII Int. Conf. "Advance in Petroleum and Gas Industry and Petrochemistry" (APGIP-7). Ukraine, Lviv 2014, 64.

[2] Alimbekova B., Korganbayeva Zh., Himersen H. et al.: J. Chem. Chem. Eng., 2014, 3, 265.

[3] Jumadilov T., Abilov Zh., Kaldayeva S. et al.: J. Chem. Eng. Chem. Res., 2014, 1, 253.

[4] Jumadilov T., Abilov Zh., Kondaurov R. et al.: Khim. Zh. Kazakhstana, 2015, 2, 79

[5] Akhylbekova M., Kondaurov R., Jumadilov T., Umerzakova M.: Khim. Zh. Kazakhstana, 2015, 4, 31.

[6] Jumadilov T., Kondaurov R., Khakimzhanov S. et al.: Khim. Zh. Kazakhstana, 2018,.1, 42

[7] Petrukhin O.: M. Praktikum po Physicokhimicheskym Metodam Analiza. Khimiya, Moskva 1987.

[8] Jumadilov T. K., Kondaurov R., Abilov Zh. et al.: Polym. Bull., 2017, 74, 4701. https://doi.org/10.1007/s00289-017-1985-3

[9] Jumadilov T., Abilov Zh., Grazulevicius J. et al.: Chem. Che. Technol., 2017, 11, 188. https://doi.org/10.23939/chcht11.02.188 [10] Suberlyak O., Mel'nyk Y., Skorokhoda V.: Mater. Sci., 2015, 50, 889. https://doi.org/10.1007/s11003-015-9798-8

[11] Grytsenko O., Suberlyak O., Moravskyi V., Gayduk A.: East-Eur. J. Enterpr. Technol., 2016, 1, 26. https://doi.org/10.15587/17294061.2016.59506

[12] Semenyuk N., Kostiv U., Suberlyak O., Skorokhoda V.: Chem. Chem. Technol., 2013, 7, 95. https://doi.org/10.23939/chcht07.01.095 [13] Jumadilov T., Abilov Zh., Kondaurov R.: Intergel systems: highly effective instruments for rare earth elements extraction from industrial solutions [in:] Mukbaniani O., Abadie M., Tatrishvili T. (Eds.), Chemical Engineering of Polymers. Production of Functional and Flexible Materials. Apple Academic Press 2017, 267-279.

[14] Jumadilov T., Yermukhambetova B., Panchenko S., Suleimenov I.: AASRI Procedia, 2012, 3, 553.

https://doi.org/10.1016/j.aasri.2012.11.087

Received: November 30, 2018 / Revised: January 15, 2019 / Accepted: June 12, 2019

\section{ФЕНОМЕН ДИСТАНЦЙНОЇ ВЗАСМОДІЇ І СОРБЦІЙНОЇ ЗДАТНОСТІ ЗШИТИХ ГІДРОГЕЛІВ ПОЛІМЕТАКРИЛОВОЇ КИСЛОТИ І ПОЛІ-4- ВІНІЛПІРИДІНУ ВІДНОСНО ЙОНІВ ЕРБІЮ}

Анотація. Досліджено вплив гідрогелю поліметакрилової кислоти-полі-4-вінілпіридину (інтергелева система gPMAA-gP4VP) на сорбиію йонів ербію. Встановлено, щзо структура основного гідрогелю має значний вплив на самоорганізаиію гідрогелю РМАА. Взаємну активаичію гідрогелів досліджено у водному середовищі. Встановлено залежності коефіцієєта набухання, питомої електропровідності та рН водних розчинів. Визначено, щчо ступінь екстракиії йонів ербію для РМАA і Р4VP становлять $44 \%$ і $17 \%$, відповідно. Показано, що при співвідношеннях 5:1 (83\% gPMAA-17\% gP4VP) $i$ 4:2 (67\% gMPAA-33\% gP4VP), були відновлені $56 \%$ і $53 \%$ йонів ербію відповідно. Через 48 год гідрогелі РМАA $і$ Р4VР мали ступінь зв'язування полімерного ланцюга $70 \%$ Спостерігались значні зміни в електрохімічних і конформаційних ступенях початкових макромолекул в інтергелевій системі gPMAA-gP4VP.

Ключові слова: інтергелева система, поліметакрилова кислота, полі-4-вінілпіридин, дистаниійна взаємодія, йони Еr ${ }^{3+}$, сорбиія. 